\title{
Omics analysis reveals the mechanism of metabolic oscillation during continuous VHG ethanol fermentation by Saccharomyces cerevisiae
}

\author{
Xue Zhang ${ }^{1}$, Liang Wang ${ }^{2}$, Qian $\mathrm{Li}^{3}$, Riaan $\mathrm{Haan}^{4}$, Fan $\mathrm{Li}^{5}$, Chen-Guang Liu ${ }^{1}$, and Fengwu \\ $\mathrm{Bai}^{1}$ \\ ${ }^{1}$ Shanghai Jiao Tong University \\ ${ }^{2}$ Dalian Polytechnic University \\ ${ }^{3}$ Dalian University \\ ${ }^{4}$ University of the Western Cape \\ ${ }^{5}$ COFCO Nutrition and Health Research Institute
}

June 15, 2020

\begin{abstract}
During continuous very high gravity (VHG) fermentation, yeast cells exhibit sustained oscillation of residual glucose, ethanol, and biomass, which remains a fundamental and unanswered question associated with product inhibition. In this study, the oscillating process was characterized through transcriptome and metabolome analysis in one sinusoid cycle. By integrating analysis of 26 metabolites and 90 genes related to carbon metabolism, the results confirmed that fermentation oscillation could be attributed to intercellular metabolic oscillation with phase difference of sinusoidal waveform. Furthermore, expression changes of stress response genes indicated that dynamic ethanol inhibition was a primary factor responsible for the oscillation of metabolism. This study not only contributes to elucidation of the mechanism of oscillating fermentation through strong product inhibition, but also provides new understanding of other fermentation processes in an unsteady state.
\end{abstract}

\section{Keywords}

Saccharomyces cerevisiae ; oscillation; ethanol inhibition; metabolomics; transcriptomics

\section{Introduction}

As the demand for oil further increases, the diversification of fuel supply through alternative energy sources such as fuel ethanol has become part of the national energy policy in many countries (Kircher, 2015). Although cellulosic ethanol is widely regarded as a promising liquid fuel (Liu et al., 2019), a lack of substantive breakthroughs in core technologies and low economic feasibility is still a major limitation. This is a result of complex pretreatment requirements, inhibitory compounds in the fermentation broth, the cost of cellulases, and inefficient pentose metabolization by the fermentation strain (Han et al., 2020). In addition, achievable cellulosic ethanol titers $(3 \%-6 \% \mathrm{v} / \mathrm{v})$ are lower than starch-based ethanol titers $(10 \%-12 \% \mathrm{v} / \mathrm{v})$ which significantly increases energy cost for distillation and stillage treatment (Bai, Anderson, \& Moo-Young, 2008).

Very high gravity (VHG) fermentation with [?]25\% w/v substrate improves ethanol titer ([?]15\% v/v) with concomitant savings in energy consumption (Puligundla, Smogrovicova, Obulam, \& Ko, 2011). VHG ethanol fermentation is usually practiced via labor-intensive and time-consuming batch operation, which is not suitable for production at large scale. On the other hand, continuous VHG ethanol fermentation (CVEF) could be applied in industry, but often trigger a sustained oscillation of residual glucose, ethanol, and biomass, 
leading to an undesirable unstable process (Bai, Ge, Anderson, \& Moo-Young, 2009). The toxicity of ethanol to the yeast $S$. cerevisiae is a primary factor limiting titer and productivity. Ethanol tolerance is related to the fluidity, structure, and composition of the plasma membrane, and the levels of unsaturated fatty acids, ergosterol, amino acids, inositol, heat shock proteins (HSPs), ATPase and the storage carbohydrates (Lam, Ghaderi, Fink, \& Stephanopoulos, 2014).

Attenuating oscillation has been attempted by engineering strategies. Employment of three tubular bioreactors in series following a stirred tank bioreactor packed with intalox ceramic saddle packing enhanced the yeast tolerance to ethanol through step-by-step adaption and cell immobilization (Bai, Chen, Anderson, \& Moo-Young, 2004). Gas stripping with nitrogen or fermentation exhaust gas directly decreased the ethanol in the broth that help yeast cell to relieve ethanol toxicity (Wang, Zhao, Xue, \& Bai, 2013). These approaches effectively attenuated oscillations and achieved quasi-steady-states in ethanol fermentation.

Periodic behavior has been investigated in eukaryotic and prokaryotic organisms (Panda, 2016). S. cerevisiae exhibits various modes of oscillatory behavior both in cell-free extract and during continuous culture (Bai et al., 2009; Chin, Marcus, Klevecz, \& Li, 2012) in the form of glycolytic oscillation with a period of about $1 \mathrm{~min}$, respiratory oscillation with a period of 40-60 min, and cell cycle oscillation with a period of 2-45 h (Richard, 2003). The glycolytic oscillation is mediated by glycolytic intermediates, and the allosteric properties of phosphofructokinase (PFK) and the positive feedback exerted on it were assumed to be responsible for periodic operation (Gustavsson et al., 2014; Olsen, Stock, \& Bagatolli, 2020). The respiratory oscillation, monitored by dissolved oxygen, oxygen uptake rate (OUR), and carbon dioxide evolution rate (CER), was modulated by sulfate assimilation, ethanol degradation and respiration (Patnaik, 2003; Tu et al., 2007). The cell cycle oscillations, synchronized with the cell division cycle, were represented by extracellular variables (OUR, CER, glucose, ethanol, and biomass), intracellular variables (storage carbohydrates), and cell cycle related variables (budding index and cell size distribution) (Ewald, Kuehne, Zamboni, \& Skotheim, 2016).

Although dynamic ethanol inhibition has been proved to be a key factor in the induction of oscillatory behavior (Wang et al., 2013), the detail of yeast cells response remains a fundamental and unanswered question. In this study, a systematic approach via transcriptomic and metabolomic analysis was carried out, with particularly focus on the cellular events of $S$. cerevisiae under CVEF and cellular metabolism affected by the fermentation parameters. A way to comprehend an unsteady state of fermentation in such a complex metabolic oscillation is demonstrated.

\section{Materials and Methods}

\section{Strain, medium, and chemicals}

All experiments were performed with an industrial yeast strain S. cerevisiae 4126. Yeast extract peptone dextrose (YPD) medium composed of $5 \mathrm{~g} / \mathrm{L}$ yeast extract powder, $3 \mathrm{~g} / \mathrm{L}$ peptone (Sangon Biotech, Shanghai) and different levels of glucose $(30,120,280 \mathrm{~g} / \mathrm{L})$ was used. YPD medium containing $30 \mathrm{~g} / \mathrm{L}$ and $120 \mathrm{~g} / \mathrm{L}$ glucose for pre-cultures in flasks and fermenters was sterilized by the usual method $\left(121^{\circ} \mathrm{C}, 20 \mathrm{~min}\right)$, while the YPD medium composed of $280 \mathrm{~g} / \mathrm{L}$ glucose for continuous ethanol fermentation was sterilized at $110^{\circ} \mathrm{C}$ for 15 min to minimize the Maillard reaction between amino acids and reducing sugars. YPD medium containing of $120 \mathrm{~g} / \mathrm{L}$ and $280 \mathrm{~g} / \mathrm{L}$ glucose was perceived as low gravity medium and very high gravity (VHG) medium, respectively. The following metabolites: GLC, G6P, F6P, FBP, 6PG, R5P, T6P, TRE, GAP, GOLP, 2+3PG, PEP, PYR, OAA, CIT, AKG, SUC, FUM, MAL, ATP, ADP, AMP, NAD ${ }^{+}, \mathrm{NADH} \mathrm{NADP}^{+}, \mathrm{NADPH}$ AcCoA (refer to the list of abbreviations) used as standards for LC-MS/MS analysis were purchased from Sigma-Aldrich.

\section{Pre-culture and continuous VHG ethanol fermentation}

Pre-cultures in $250 \mathrm{~mL}$ shake-flasks and 2.5-L laboratory stirred tank reactor (KoBioTech, Korea) with a working volume of $1.7 \mathrm{~L}$ were cultured at $30^{\circ} \mathrm{C}$ and $300 \mathrm{rpm}$. The $\mathrm{pH}$ and aeration rate were controlled at 4.50 (adjusted with $2 \mathrm{~mol} / \mathrm{L}$ sterilized $\mathrm{NaOH}$ ) and $0.05 \mathrm{vvm}$ for the bioreactor. When residual glucose in the pre-culture was below $1.0 \mathrm{~g} / \mathrm{L}, \mathrm{CVEF}$ operation was initiated at the same conditions as above. The VHG 
medium was fed for continuous ethanol fermentation at a dilution rate of $0.027 \mathrm{~h}^{-1}$.

\section{Fermentation parameters determination}

Fermentation parameters containing the concentrations of residual glucose, ethanol, glycerol, succinate, biomass, cell viability, and oxidation reduction potential (ORP) were investigated to assess the CVEF system.

Triplicate samples were withdrawn and centrifuged at 10,000 rpm at ambient temperature for $5 \mathrm{~min}$. The cell pellet was collected for biomass determination, and the supernatant was frozen at $-20 \mathrm{degC}$ for HPLC analysis. An HPLC system with RI-detector (Waters 410, Waters, USA) and an Aminex HPX 87-H column (300 x7.8 mm, Bio-Rad Laboratories, Hercules, CA, USA) was used to analyze glucose, ethanol, glycerol, and succinate in the effluent according to Wang et al.(2013). Biomass was measured as dry cell weight (DCW), wash the cell pellet obtained previously twice with deionized water, overnight at $80 \mathrm{degC}$ to constant weight, and weigh the DCW. Cell viability was evaluated via methylene blue stain described by Bai et al. (2004). Living cells were calculated from biomassxviability. ORP value was monitored by an ORP electrode.

\section{Sampling and extraction for extracellular metabolites and total RNA}

For the sampling of total RNA and extracellular metabolites in yeast cells, approximately 5-mL sample per time interval was rapidly withdrawn from the fermenter. Cell pellets were immediately harvested by centrifugation at ambient temperature and 10,000 rpm for $2 \mathrm{~min}$, washed with $0.9 \%(\mathrm{w} / \mathrm{v}) \mathrm{NaCl}$, centrifuged at ambient temperature and 10,000 rpm for $2 \mathrm{~min}$, and then frozen at $-80 \mathrm{deg}$ for further analysis. The supernatant was frozen at $-20 \operatorname{deg} C$ for determination of extracellular metabolites.

Total RNA was extracted by the RNeasy Mini Kit (Qiagen, Germany). RNA quality was confirmed by $\mathrm{A}_{260} / \mathrm{A}_{280}$ ratio (>2.0) and agarose gel electrophoresis (28S:18S ratio=1.5-2.0). RNA samples were dissolved in Milli-Q water and frozen at $-80 \mathrm{deg} C$ for transcriptome analysis.

\section{Transcriptomic analysis}

High-resolution genome-wide transcriptional analysis with RNA-Seq was carried out by Beijing Genomics Institute (BGI-Huada, Shenzhen, China). Raw reads were filtered to remove some adapters and low-quality reads, and the remaining reads were mapped to the reference genome S288c_reference_genome_R6-1-1_20110203 to get the transcripts information. Next, the expression levels of the differentially expressed genes (DEGs) were normalized by RPKM, defined as the number of reads per kilobase per million mapped

reads. To determine the DEGs in different periods, absolute value of $\log _{2}$-fold changes larger than 1 and false discovery rate lower than 0.01 were selected as screening criteria.

\section{Confirmation of transcriptomic results by qRT-PCR}

To determine the copy numbers of the transcripts, quantitative real-time PCR (qRT-PCR) was conducted for each sample. Six representative genes, ZPS1 (zinc regulatory proteins), DSE1 (cell division), HPF1 (mannose glycoprotein), EGT2 (cell division),TIS11 (iron regulatory proteins) and, GCV1 (synthesis of coenzyme factors) were selected to perform the verification. The primers are shown in Table S1.

\section{Quenching and extraction of intracellular metabolites}

Approximately $5 \mathrm{~mL}$ sample was rapidly taken from fermenter and injected into $25 \mathrm{~mL}$ precooled (-40degC) $60 \%(\mathrm{v} / \mathrm{v})$ methanol solution to interrupt the cell metabolism as soon as possible, resulting in a final concentration of $50 \%(\mathrm{v} / \mathrm{v})$ methanol. The quenching process lasted 3 min. Cell pellets were then separated using a fast vacuum dependent filtration $(0.45 \mu \mathrm{m}$ nominal pore size $)$ and washed with $5 \mathrm{~mL}$ precooled $\left(-40^{\circ} \mathrm{C}\right)$ $50 \%(\mathrm{v} / \mathrm{v})$ methanol solution to remove the residual medium component on the cell surface. Next the filter adhered with cell pellet was frozen by liquid nitrogen, and rapidly transferred into $9.5 \mathrm{~mL}$ precooled $\left(-40^{\circ} \mathrm{C}\right)$ extraction solution composed of $2.5 \mathrm{~mL}$ methanol, $5 \mathrm{~mL}$ chloroform, and $2 \mathrm{~mL}$ fresh Tricine-EDTA buffer solution $\left(3 \mathrm{mmol} / \mathrm{L}\right.$ Tricine and $3 \mathrm{mmol} / \mathrm{L} \mathrm{EDTA}$, $\mathrm{pH} 7.0$, stored at $\left.4^{\circ} \mathrm{C}\right)$. The mixture was vigorously vibrated for $60 \mathrm{~min}$ at $-40 \pm 5^{\circ} \mathrm{C}$ (dry ice-ethanol bath). The first aqueous phase was collected by a centrifugation step $\left(12,000 \mathrm{rpm}, 5 \mathrm{~min},-19^{\circ} \mathrm{C}\right)$, more methanol and Tricine-EDTA buffer solution was added in for a second 
extraction, and the second aqueous phase was collected and pooled with the first one. The aqueous phase was stored at $-80 \mathrm{degC}$ and evaporated to dryness before use. The dry residue was reconstituted in $100 \mu \mathrm{L}$ of Mill-Q water for LC-MS/MS analysis.

\section{LC-MS/MS analysis}

LC instrumentation included a high-performance liquid chromatography integrated system (Thermo Fisher Scientific Inc., Waltham, MA, USA) with a XBridge BEH Amide column $(2.5 \mu \mathrm{m}, 2.1 \times 100 \mathrm{~mm}$, Waters $)$ at $25^{\circ} \mathrm{C}$. MS experiments were performed using a triple quadrupole mass spectrometer TSQ Quantum UltraTM (Thermo Fisher Scientific Inc., Waltham, MA, USA). The auto-sampler temperature was kept at $4^{\circ} \mathrm{C} .5 \mu \mathrm{L}$ of sample was injected with the full loop mode into the system. The XBridge BEH Amide column was used under acidic conditions, and the mobile phase consisted of two eluents: eluent $\mathrm{A}$ was $20 \mathrm{mmol} / \mathrm{L} \mathrm{NH}_{4} \mathrm{Ac}$ in Milli-Q water, pH 6.5 and eluent B was $100 \%$ acetonitrile. A gradient was run at a flow rate of $0.3 \mathrm{~mL} / \mathrm{min}$; it started with $5 \% \mathrm{~A}$, which was increased linearly to $60 \% \mathrm{~A}$ in 20 min and stayed at $60 \% \mathrm{~A}$ for 5 min, then decreased to $5 \% \mathrm{~A}$ in $10 \mathrm{~min}$. An equilibration time of $15 \mathrm{~min}$ was allowed before the next injection.

Ionization was performed using an electrospray ionization source (ESI) operating in the positive and negative ionization modes. Sheath and auxiliary gas was nitrogen. The collision gas was argon. Xcalibur 2.2 software (Thermo Fisher Scientific Inc.) was used for instrumental control and data acquisition. Eighteen compounds presented greater ionization efficiency in negative than in positive ionization mode (namely G6P, F6P, FBP, 6PG, R5P, T6P, TRE, GAP, GOLP, 2+3PG, PEP, PYR, OAA, CIT, AKG, SUC, FUM and MAL), while for eight compounds the positive mode improved ionization efficiency (namely ATP, ADP, AMP, NAD ${ }^{+}$, $\mathrm{NADH}, \mathrm{NADP}^{+}, \mathrm{NADPH}$ and $\mathrm{AcCoA}$ ). Selected reaction monitoring mode was set, and the collision energy for an effective fragmentation of the selected precursor ions was optimized according to the analyte. The spray voltage, vaporizer temperature, sheath gas pressure, collision pressure, and aux gas pressure were 2.5 $\mathrm{kV}, 250{ }^{\circ} \mathrm{C}, 35 \mathrm{psi}, 1.5 \mathrm{psi}$, and $10 \mathrm{psi}$, respectively.

\section{Results}

\section{Physiology of S. cerevisiae in continuous VHG ethanol fermentation}

During CVEF, oscillatory behavior of fermentation parameters, such as biomass, cell viability, ethanol, residual glucose, and glycerol, are displayed periodically and sustained with a period of approximately $150 \mathrm{~h}$ at the dilution rate of $0.027 \mathrm{~h}^{-1}$ (Bai, Chen, Anderson, et al., 2004; Wang et al., 2013). In order to focus on the intracellular regulation mechanism of yeast cells during the oscillatory process, a complete representative period was shown in Figure 1, in which ethanol, glycerol, biomass, cell viability and living cells exhibited the similar changing tendencies, while residual glucose presented the opposite profile.

Considering the periodic wave characteristics, the different phases of sinusoidal function $(0 \pi, \pi / 2, \pi$, and $3 \pi / 2)$ are appropriately applied to describe the changing trends of parameters for better exploring and comprehending their correlations (Figure S1). The oscillation curve of residual glucose is configured as a $0 \pi$ phase, so the profiles of ethanol and glycerol are correspondingly considered as a $\pi$ phase. Because ethanol as the major product requires the consumption of glucose in proportion, it naturally follows that there is an opposite tendency between them. The parameters associated with cell growth (biomass, cell viability, and living cells) are also classified as a $\pi$ phase based on the foundational knowledge that cell growth couples with the generation of primary metabolites such as ethanol. However, the changes of cell growth and viability happened prior to other parameters, reflecting a delayed response of intracellular metabolites. In addition, both high glucose and ethanol caused a synergy inhibition on viability. According to the biomass curve, five representative sampling points (P1-5 in Figure 1) were selected for subsequent metabolic profile analysis and transcriptomic analysis.

\section{Statistical analysis of DEGs}

RNA transcription data was integrated and intercompared in Figure 2. It is obvious that cellular transcriptional response of yeast cells at P1 was consistent with that at P5 - only 10 DEGs detected, which suggest that yeast cells exhibit similar transcriptional characteristics in the same position of different oscillation 
periods. This was expected as the end point of one oscillation represents the start point of the following period. It also confirmed the periodic nature of continuous oscillation.

The big DEGs counts between the neighboring points showed the sustained and significant transcriptional changes attributed to the unsteady state of VHG ethanol fermentation. Compared to initial cell status (P1), the curve of total counts of DGEs in P2-5 fitted the biomass curve ( $\pi$ phase). The highest counts of DEGs appeared between $\mathrm{P} 1$ and $\mathrm{P} 3$ due to the maximum time difference of $\mathrm{P} 3$ and $\mathrm{P} 1$ (or P5 that is equivalent to $\mathrm{P} 1)$.

The mRNA expression of six selected genes (upregulated during P1-P2-P3 and downregulated during P3-P4P5) was measured by qRT-PCR. These genes are common differential genes compared by several adjacent points. The trend of gene expression levels in five sampling points of 6 DEGs were consistent with that of RNA-seq, which verified the accuracy of the DEGs from transcriptome analysis (Figure S2).

\section{Intermediates and gene expression associated with central carbon metabolism}

Cells are able to assess the magnitude of metabolic flux by tuning the rate of metabolic reactions, consequently regulating cellular physiology under various stressful conditions (Litsios, Ortega, Wit, \& Heinemann, 2018). During CVEF with glucose as the sole carbon source and major products such as ethanol, $\mathrm{CO}_{2}$, biomass, and glycerol, the central carbon metabolism pathways of $S$. cerevisiae cells mainly constitute the EmbdenMeyerhof-Parnas pathway (EMP), the pentose phosphate pathway (PPP) and the tricarboxylic acid cycle (TCA) (Figure S3). Thus, metabolomics and transcriptomic datasets of yeast were integrated and interpreted via a pathway-based method, referring to 26 metabolites and 90 genes related to central carbon metabolism, whose responses in an oscillation period were visualized in Figure 3.

Glucose firstly fluxes into EMP to provide ATP and precursors for cell growth. EMP can be divided into an energy-consuming phase ( $-2 \mathrm{~mol} \mathrm{ATP} / \mathrm{mol}$ glucose from $\mathrm{GLC}_{\text {in }}$ to GAP) and an energy-producing phase (4 mol ATP/mol glucose from GAP to PYR). Most of the pyruvate produced is converted into ethanol in the condition of low dissolved oxygen. The PPP as the source of NADPH and pentose contains an oxidative pathway (from G6P to Ri5P) and a non-oxidative pathway (from Ri5P to F6P and GAP). The TCA is a series of enzyme-catalyzed chemical reactions that form a key part of aerobic respiration in cells. As to the changing tendency of carbon metabolism during the oscillation (Figure 3), it was weakened during the P2 to P3 phase (especially the EMP and TCA processes) while being enhanced during the P3 to P5 with the decreasing residual glucose and increasing ethanol concentration.

The significant decline of most metabolites with associated down-regulated gene expression was observed in $\mathrm{P} 3 / \mathrm{P} 2$, but the opposite phenomenon happened in $\mathrm{P} 5 / \mathrm{P} 4$. The reason may be found in Figure 1 where biomass increased $3.3 \mathrm{~g} / \mathrm{L}$ from P2 to P3, but decreased $3.32 \mathrm{~g} / \mathrm{L}$ from P4 to P5 was observed. In addition, more changed metabolites and gene expression appeared in P3/P2 and P5/P4 than that observed for P2/P1 and P4/P3 and this may be due to the biomass difference between the adjacent samples. From Figure 1, the biomass changes between P2 and P3, P4 and P5 were about two-fold of that between P1 and P2, P3 and $\mathrm{P} 4$. The pool of cofactors and ATP was enhanced in P2/P1 and P5/P4, but decreased from P2 to P4, which reflected the activity of the energy metabolic reactions. In spite of an expected sinusoidal oscillation found in Figure 3, there are many additional details that can be elucidated by analyzing the metabolites and genes one by one (Figure 4).

Figure 4A showed the specific concentration and changing trend of each metabolite, most of which are involved in Figure 3, except adenylate energy charge (EC). Throughout the oscillation cycle, ATP was the dominant intracellular adenine nucleotide and the adenylate energy charge $\left(\mathrm{EC}=\left([\mathrm{ATP}]+0.5^{*}[\mathrm{ADP}]\right) /([\mathrm{ATP}]\right.$ $+[\mathrm{ADP}]+[\mathrm{AMP}]))$ remained high $(>0.9)$, which indicates that the cell has a strong potential for phosphoryl transfer and works as the 'normally metabolizing' cell during the CVEF. In addition, we also found that the changing tendency of EC is identical with biomass (Figure 1), which seems to indicate that a higher EC value is benifitial for cell proliferation. As described in Figure S1, most profiles of genes and metabolites are able to be divided into four patterns of sin function: $0 \pi, \pi / 2, \pi, 3 \pi / 2$ phases. As shown in Figure $4 \mathrm{~B}$, the changing tendencies of 16 out of 22 genes related to EMP pathway presented a $\pi / 2$ phase, while the rest 
five genes (HXK1, HXT1,2,4 and PFK1 ) showed a $3 \pi / 2$ phase, $0 \pi$ phase and $\pi$ phase, respectively. This inferred that the processes of glucose transport, glucose phosphorylation, and the catalysis of PFK could be the nodes for the oscillation regulation of intracellular metabolites. Genes PFK1 and PFK2 encoded the $4 \alpha$ - and $4 \beta$-subunits which compose the heterooctameric enzyme(PFK)in yeast and both types of subunits contributing equally to catalysis instead of that one of the subunits functioning preferentially in allosteric regulation (Heinisch, Boles, \& Timpel, 1996). It's interesting that PFK2 was not induced more drastically between P2 and P3/4 as PFK1 was (Figure 4B), which seems to indicate that PFK1 is upregulated more rapidly and variably than $P F K 2$ in response to the increased substrate concentration. In addition, PFK activity is subject to allosteric control mechanism, with inhibition by ATP and activation by AMP (Heinisch et al., 1996). Here, the change trend of ATP (0 $\pi$ phase) is contrary to PFK1 ( $\pi$ phase), which reflects its inhibitory effect on PFK allosteric control. But the change trend of activator AMP is not consistent with PFKs, probably because its absolute oscillation change values are much lower than that of ATP, making its effect was not shown intuitively in the overall trend of PFKs. Two distinct changing tendencies for the upper and down neighboring metabolites of PFK, G6P and F6P are in $\pi / 2$ phase while FBP and GAP are in $0 \pi$ phase. A margin of $\pi / 2$ phase between their profiles was assumed to be induced by PFK activity to catalyze F6P to produce FBP, indicating that PFK is the key rate-limiting enzyme in the energy-consuming phase of the glycolytic pathway. This description is similar to the mechanism proposed for the metabolic oscillation in continuous culture or yeast extracts (Boiteux, Goldbeter, \& Hess, 1975; Papagiannakis, Niebel, Wit, \& Heinemann, 2017; Thoke et al., 2018).

In ethanol production after the EMP pathway, the RPKM value of $A D H_{4}$ was always over 2000, peaking at P3 with a value of 4300 , while $P D C 1$ and $A D H 1$ presented a $\pi / 2$ phase, consistent with most genes of the EMP pathway, contrary with $P D C 5,6$ and $A D H_{4}$ (Figure 4B). The expression profiles of $A D H_{4}$ and PDC5 are $\pi / 2$ phase earlier than ethanol, and the overall expression level of $A D H_{4}$ is much higher than that of $P D C 5$ , suggesting that Adh4p might be responsible for ethanol formation during CVEF. The gene $\mathrm{ADH}_{4}$ encodes the zinc-dependent alcohol dehydrogenase isoenzyme, and its transcription is induced in response to zinc deficiency, which was consistent with the changing tendency of the expression level of zinc deficiency related gene ZPS1 (Figure S2). The expressions of $A D H 1$ and $P D C 1$ were regulated by the same regulatory factors, while that of $A D H_{4}$ and $P D C 5$ and $P D C 6$ by a different kind. It could be assumed that the regulation of the expression of gene $A D H 1$ and $P D C 1$ were closely related to the flux changes of catabolism, while the regulation of the expression of gene $A D H_{4}$ and $P D C 5$ and $P D C 6$ were closely related to the flux changes of anabolism and zinc level.

In addition, yeast cells produced 10.2-13.7 g/L glycerol during CVEF, and the changing tendency of the extracellular glycerol level showed a $\pi$ phase pattern, which was similar to PFK1 but contrary to the GPD1, HOR2 and the ORP value (Figure S4), while those of GPD2 and RHR2 showed $\pi / 2$ phase pattern (Figure 4B). The expression of GPD1 and GPD2 are regulated by osmotic stress and redox homeostasis, and studies have shown that the HOG pathway where the GPD1 and GPD2 are located can respond to ethanol stress (Klein, Swinnen, Thevelein, \& Nevoigt, 2017; Udom, Chansongkrow, Charoensawan, \& Auesukaree, 2019), suggesting that the glycerol production during VHG oscillation was induced by osmotic stress and the redox environment.

The expression level of genes related to PPP was far below that in the EMP (Figure 4B), suggesting that cellular anabolism might be repressed during CVEF. The oxidation stage of the PPP pathway is from G6P to $6 \mathrm{PG}$, and finally Ri5P. Both steps generate NADPH, which provides reducing power and precursor metabolites for the cell's biosynthesis pathways. The changing tendency of $6 \mathrm{PG}$ showed $\pi$ phase pattern, similar to biomass (Figure 1), indicating that yeast cell growth is closely related to NADPH synthesis. Furthermore, the expression level of $A L D 4, A L D 6, M A E 1$ (Figure S5), and $A D H_{4}$ exhibited a $3 \pi / 2$ phase pattern, ahead of NADH and NADPH. The products of $A L D 4, A L D 6$ and $M A E 1$ are involved in catalyzing cofactor $\mathrm{NAD}(\mathrm{P}) \mathrm{H}$ generation steps, suggesting that cofactor $\mathrm{NAD}(\mathrm{P}) \mathrm{H}$ generation might be the driving force of PPP flux and biomass accumulation.

As to the TCA cycle, in this study, CVEF was conducted with aeration of only $0.05 \mathrm{vvm}$, therefore, the 
metabolites fluxed into the TCA cycle might be at low concentration, which was confirmed by transcriptional analysis result that showed the expression levels of genes related to TCA cycle were much lower than these related to EMP pathway.

\section{Dynamics of gene expression associated with stress response}

Yeast cells suffer from multiple stresses during CVEF, such as ethanol inhibition, osmotic stress by high residual glucose, and redox stress from imbalanced energy metabolism (Auesukaree, 2017; Burphan et al., 2018; Caspeta, Castillo, \& Nielsen, 2015). The metabolism of storage carbohydrates, such as trehalose, glycogen, and glycan, was investigated to help determine tolerance to environmental stress (Babazadeh et al., 2017; Li et al., 2009). During the oscillation, significant changes were observed for the expression level of genes related with stress response, such as oxidative stress response related genes SOD1, SOD2, CTT1, OYE2 and GRX8, and the HSP family (HSP10,12,26,31,42,60,82 ). As shown in Figure 5, it was difficult to draw a unified change rule for all, but most of the stress response genes were highly expressed at P4, when ethanol levels kept increasing in the broth and was about to achieve the maximum value. At the same time, the glucose level approached its minimum and ORP value was at an intermediate level, thus those two factors could not exert severe stresses on cells, suggesting that a large proportion of cellular response to stresses were triggered by ethanol inhibition in $\mathrm{P} 4$.

HSPs are a highly conserved family of molecular chaperone proteins that play a role in the aggregation, assembly, transport, and folding of proteins (Muhlhofer et al., 2019). Due to the common effect of multiple stresses during CVEF on disturbing protein structures, the upregulation of HSPs is observed upon exposures to all of these stresses, making the expression of HSPs overall higher. HSP10 and HSP60 are mitochondrial matrix co-chaperonin and chaperone ATPase, which cooperates with each other, so their expression levels are similar.HSP26 and HSP 42 are small heat shock proteins that form oligomers to suppress unfolded proteins aggregation. The expression levels of these HSPs are consistent with changes in ethanol concentration, indicating that cellular protein denaturation was triggered by ethanol stress.

In addition, it seems that the ethanol oscillation was mainly responsible for the expression changes of SOD1 . During the VHG fermentation, S. cerevisiae exhibited increased levels of intracellular Reactive Oxygen Species (ROS) and oxidative damage to cell structures. Superoxide dismutases (SODs), the major ROS-scavenging enzymes, are classified into two groups according to their subcellular localization and metal cofactors, namely cytosolic Cu/Zn-SOD (Sod1p) and mitochondrial Mn-SOD (Sod2p). Although both SOD1 and SOD2are highly expressed in the whole oscillation for the tolerances to ROS stress, the average and the variance of SOD1 expression is $21.6 \%$ and $53.7 \%$ higher than that of SOD2. This may be due to the production of ethanol in the cytoplasm, which brings greater ROS stress to this cellular compartment.

Although ethanol is a major stress trigger, we have also found additive effects of multiple stress conditions on the expression of some stress genes, making these genes highly expressed during the entire oscillation cycle, especially at P3 and P4 to cope with the oxidative stress caused by high osmotic pressure and strong ethanol inhibition. For example, the oxidative stress response gene OYE2 (NADPH dehydrogenase) and the glutathione-independent methylglyoxalase gene HSP31 were initially up-regulated in response to the osmotic stress caused by high glucose concentration (P2/P1), were subsequently further up-regulated due to osmotic pressure and ethanol stress (P3/P2) and finally expressed at its highest level at P4 due to strong ethanol stress.

\section{Dynamics of gene expression associated with anabolism}

Biomass as an important process parameter oscillated periodically in CVEF, indicating that the anabolism of yeast cells may also experience the changing cycle. Transcriptome analysis showed that a large amount of genes related to ribosome synthesis were also expressed in a periodic pattern. As shown in Figure 6, most DEGs of yeast cells at P2 were up-regulated in comparison with P1, and also in the comparison of gene expression of yeast cells at P5 to P4, while the DEGs of yeast cells at P4 were down-regulated compared with P3. However, there was no significant difference between the gene expression of yeast cells at P2 and P3. Further, the overview of changes in expression of the ribosome synthesis genes were presented as the 
non-standard $0 \pi$ phase pattern that only decreased in $\pi / 2$ period, not in the $\pi$ period. It was indicated that ribosome synthesis was robust in the $3 \pi / 2$ period of the oscillation (the phase from $\mathrm{P} 4, \mathrm{P} 1, \mathrm{P} 2$ to $\mathrm{P} 3$ ), while much lower in the remaining $1 / 4$ oscillation period (the phase from P3 to P4).

The up-regulated expression of genes related to ribosome synthesis symbolized the start of anabolism. Thus, it can be speculated that cell anabolism was initiated at P4, and continued to P3 (P4-P5(P1)-P2-P3, 3/4 oscillation period), while the biomass presented as the $\pi$ phase pattern that exist about $\pi$ phase later than the ribosome synthesis. The increased expression of ribosomal genes prior to biomass accumulation indicated that ribosome synthesis was a material preparation process during the interval of cell division and that the genes related to ribosome synthesis were prioritized and expressed. In addition, the periodic change of ribosomal synthesis gene were opposite to the ethanol concentration, and there was a $\pi$ phase difference between them, which may suggest that the regulation of ribosomal synthesis during VHG oscillation is also related to the ethanol stress.

\section{Discussion}

Transcriptomic and metabolomic analysis were employed in this study to address the cellular response of yeast cells during sustained VHG oscillation. In a typical sinusoid period, P1 was consistent with P5 due to almost no difference in gene expression observed between them. Thus, the VHG oscillation is regarded as a closed cycle. The end point P5 is essentially just the start point P1 for the next period.

Among all kinds of stresses that yeast cells encounter in CVEF, dynamic ethanol inhibition was highlighted in previous studies and recognized to be responsible for oscillation(Wang et al., 2013). Expression level of most genes referring to anabolism, such as ribosome synthesis pathway, were up-regulated from phase P1 to P3, while down-regulated from phase P3 to P5, presented as an $0 \pi$ phase pattern, suggesting that cellular anabolism might be regulated by the similar factors. Further, combined with the changing of ethanol, which presented as a $\pi$ phase pattern, this suggests that cellular anabolism might be modulated by ethanol inhibition.

A certain critical ethanol concentration ( $\mathrm{E}_{\text {crit }}$ ) was speculated at approximately $50 \mathrm{~g} / \mathrm{L}$ (Wang et al., 2013). If the ethanol concentration exceeded the $\mathrm{E}_{\text {crit }}$, the cell proliferation of the yeast culture was significantly inhibited while at the same time the biomass level was decreasing due to continuous dilution. However, most cells still survived and produced ethanol without cell proliferation, thus the ethanol in the broth would climb gradually to the maximum concentration until yeast cells lost fermentation ability. Then ethanol in the broth would decrease due to continuous dilution, until the ethanol inhibition imposed on yeast cells was attenuated. When the ethanol concentration declined below $\mathrm{E}_{\text {crit }}$, the yeast cells would gradually revive the abilities of proliferation and fermentation, such as ribosome synthesis and the biomass and ethanol levels in the broth accumulated gradually. Unless the specific rate of ethanol production and biomass formation passed over the dilution rate $\left(\mathrm{D}=0.027 \mathrm{~h}^{-1}\right)$, biomass and ethanol in broth would decrease for a long time. As soon as the yeast cells proliferated and the ethanol production rate surpassed the dilution rate, the ethanol concentration increased to a higher level. The ethanol inhibition imposed on yeast cells would then become stronger and cell proliferation and fermentation would subsequently be inhibited and another VHG oscillation period would be initiated. Hence, the sustained VHG oscillation is a result of the competition of cell growth, ethanol formation and ethanol inhibition.

The concentration of metabolites in the glycolytic pathway exhibited a cyclical oscillation behavior. There was a $\pi / 2$ phase difference between the curve of metabolites in the energy consumption stage and the production stage. The generation of this oscillation behavior may originate from phosphofructokinase (PFK), indicating that the VHG oscillation behavior may be the result of the combined effect of intracellular regulation and extracellular ethanol stress in yeast cells.

This work has analyzed the intracellular responses of the yeast cells to the dynamic ethanol inhibition during VHG oscillation. The enhancement of ethanol tolerance of the strain could alleviate the stress of ethanol on cells, thus helping to attenuate the oscillation effect in CVEF. In addition, further transformation of related metabolic pathways by genetic engineering may weaken the oscillation behavior at the cellular molecular 
level, which may enable VHG continuous ethanol fermentation technology to meet industrial production requirements.

\section{Conclusions}

Continuous ethanol fermentation using VHG substrate by $S$. cerevisiae in a stirred tank reactor was carried out in this study, and oscillatory behavior of fermentation parameters was exhibited periodically and in a sustained manner with a period of approximately $130 \mathrm{~h}$. Here, we also analyzed the intracellular oscillation behavior and demonstrated that there was a lag response between macro-parameters, extracellular metabolites, and the expression level of genes. In central carbon metabolism, the regulation of PFK was shown pointed to play an important role in the regulation of the oscillations of glycolytic metabolites. For ethanol formation, it was assumed that the regulation of the expression of gene $A D H 1$ and PDC1 was closely related to the flux changes of catabolism, while the regulation of the expression of genes $A D H_{4}, P D C 5$ and $P D C 6$ was closely related to the flux changes of anabolism. Glycerol was formed due to both osmotic stress response and redox homeostasis mechanisms, but the flux of the TCA cycle was negligible during CVEF at low oxygen-supplemented conditions. Oxidative stress response related genes and the HSPs family genes were observed to be significantly changed and mainly triggered by ethanol inhibition. In anabolic process, the ribosome synthesis genes were prioritized and expressed before the biomass change, suggesting that it needs time for cell construction. Finally, we proposed a mechanism to describe the oscillatory process, and the sustained VHG oscillation is considered as a result of the competition of cell growth, ethanol formation and ethanol inhibition.

\section{Acknowledgements}

We appreciate financial support from National Key R\&D Program of China from Ministry of Science and Technology of the People's Republic of China [grant number 2018YFB1501401], National Natural Science Foundation of China [grant number 31500040, 21978167], and the Natural Science Foundation of Shanghai [grant number 18ZR1420700, 19160745300]. We thank Prof. Hua Zhang and Dr. Huihui Wan in State Key Laboratory of Fine Chemicals, Dalian University of Technology for the metabolic analysis.

\section{Abbreviations}

\begin{tabular}{ll}
\hline $1,3-B P G$ & 1,3 -bis-Phospho-Glycerate \\
\hline $2+3 P G$ & 2 -+3-Phosphoglycerate \\
6 PG & 6-Phosphogluconate \\
6 PGL & 6-Phospho-glucono-1,5-lactone \\
AcCoA & Acetyl-CoA \\
ACE & Acetate \\
AKG & $\alpha$-Ketoglutarate \\
ALD & Aldehyde dehydrogenase \\
CER & Carbon dioxide evolution rate \\
CIT CVEF & Citrate Continuous VHG ethanol fermentation \\
DCW & Dry cell weight \\
DEGs & Differentially expressed genes \\
DHAP & Di-OH-acetone-phosphate \\
E4P & Erythrose-4-phosphate \\
EC EMP & Energy charge Embden-Meyerhof-Parnas \\
ETH & Ethanol \\
F6P & Fructose-6-phosphate \\
FBP & Fructose-1,6-diphosphate \\
FUM & Fumarate \\
G1P & Glucose-1-phoshate \\
G3P & 3-Phosphoglycerate \\
G6P & Glucose-6-phoshate
\end{tabular}




\begin{tabular}{ll}
\hline $1,3-$-BPG & 1,3-bis-Phospho-Glycerate \\
\hline GAP & Glyceraldehyde-3-phosphate \\
GLC & Glucose \\
GOL & Glycerol \\
GOLP & Glycerol-3-phosphate \\
HThPP & 2-Hydroxyethyl-ThPP \\
HSPs & Heat shock proteins \\
ICIT & Isocitrate \\
MAL & Malate \\
OAA & Oxaloacetate \\
ORP & Oxidation reduction potential \\
OUR & Oxygen uptake rate \\
PEP & Phosphoenolpyruvate \\
PFK & Phosphofructokinase \\
PPP & pentose phosphate pathway \\
PYR & Pyruvate \\
R5P & Ribose-5-phosphate \\
Ri5P & Ribulose-5-phosphate \\
RNA-Seq RPKM ROS & RNA sequencing Reads per kilobase per million mapped reads Reactive Oxygen Species \\
SUC & Succinate \\
SuCoA & Succinyl CoA \\
T6P & Trehalose-6-phosphate \\
TRE & Trehalose \\
VHG & Very high gravity \\
\hline
\end{tabular}

\section{References}

Auesukaree, C. (2017). Molecular mechanisms of the yeast adaptive response and tolerance to stresses encountered during ethanol fermentation. Journal of Bioscience and Bioengineering, 124 (2), 133-142. doi:10.1016/j.jbiosc.2017.03.009

Babazadeh, R., Lahtvee, P. J., Adiels, C. B., Goksor, M., Nielsen, J. B., \& Hohmann, S. (2017). The yeast osmostress response is carbon source dependent. Scientific Reports, 7 (1), 990. doi:10.1038/s41598-01701141-4

Bai, F. W., Anderson, W. A., \& Moo-Young, M. (2008). Ethanol fermentation technologies from sugar and starch feedstocks. Biotechnology Advances, 26 (1), 89-105. doi:10.1016/j.biotechadv.2007.09.002

Bai, F. W., Chen, L. J., Anderson, W. A., \& Moo-Young, M. (2004). Parameter oscillations in a very high gravity medium continuous ethanol fermentation and their attenuation on a multistage packed column bioreactor system. Biotechnology and Bioengineering, 88 (5), 558-566. doi:10.1002/bit.20221

Bai, F. W., Chen, L. J., Zhang, Z., Anderson, W. A., \& Moo-Young, M. (2004). Continuous ethanol production and evaluation of yeast cell lysis and viability loss under very high gravity medium conditions. Journal of Biotechnology, 110 (3), 287-293. doi:10.1016/j.jbiotec.2004.01.017

Bai, F. W., Ge, X. M., Anderson, W. A., \& Moo-Young, M. (2009). Parameter oscillation attenuation and mechanism exploration for continuous VHG ethanol fermentation.Biotechnology and Bioengineering, 102 (1), 113-121. doi:10.1002/bit.22043

Boiteux, A., Goldbeter, A., \& Hess, B. (1975). Control of oscillating glycolysis of yeast by stochastic, periodic, and steady source of substrate: a model and experimental study. Proc Natl Acad Sci U S A, 72 (10), 3829-3833. doi:10.1073/pnas.72.10.3829 
Burphan, T., Tatip, S., Limcharoensuk, T., Kangboonruang, K., Boonchird, C., \& Auesukaree, C. (2018). Enhancement of ethanol production in very high gravity fermentation by reducing fermentation-induced oxidative stress in Saccharomyces cerevisiae. Scientific Reports, 8 (1), 13069. doi:10.1038/s41598-018$31558-4$

Caspeta, L., Castillo, T., \& Nielsen, J. (2015). Modifying Yeast Tolerance to Inhibitory Conditions of Ethanol Production Processes. Frontiers in Bioengineering and Biotechnology, 3 , 184. doi:10.3389/fbioe.2015.00184

Chin, S. L., Marcus, I. M., Klevecz, R. R., \& Li, C. M. (2012). Dynamics of oscillatory phenotypes in Saccharomyces cerevisiae reveal a network of genome-wide transcriptional oscillators. FEBS Journal, 279 (6), 1119-1130. doi:10.1111/j.1742-4658.2012.08508.x

Ewald, J. C., Kuehne, A., Zamboni, N., \& Skotheim, J. M. (2016). The Yeast Cyclin-Dependent Kinase Routes Carbon Fluxes to Fuel Cell Cycle Progression. Molecular Cell, 62 (4), 532-545. doi:10.1016/j.molcel.2016.02.017

Gustavsson, A. K., van Niekerk, D. D., Adiels, C. B., Kooi, B., Goksor, M., \& Snoep, J. L. (2014). Allosteric regulation of phosphofructokinase controls the emergence of glycolytic oscillations in isolated yeast cells. FEBS Journal, 281 (12), 2784-2793. doi:10.1111/febs.12820

Han, C., Yang, R., Sun, Y., Liu, M., Zhou, L., \& Li, D. (2020). Identification and Characterization of a Novel Hyperthermostable Bifunctional Cellobiohydrolase- Xylanase Enzyme for Synergistic Effect With Commercial Cellulase on Pretreated Wheat Straw Degradation. Frontiers in Bioengineering and Biotechnology, 8 , 296. doi:10.3389/fbioe.2020.00296

Heinisch, J. J., Boles, E., \& Timpel, C. (1996). A yeast phosphofructokinase insensitive to the allosteric activator fructose 2,6-bisphosphate. Glycolysis/metabolic regulation/allosteric control. Journal of Biological Chemistry, 271 (27), 15928-15933. doi:10.1074/jbc.271.27.15928

Kircher, M. (2015). Sustainability of biofuels and renewable chemicals production from biomass. Current Opinion in Chemical Biology, 29 , 26-31. doi:10.1016/j.cbpa.2015.07.010

Klein, M., Swinnen, S., Thevelein, J. M., \& Nevoigt, E. (2017). Glycerol metabolism and transport in yeast and fungi: established knowledge and ambiguities. Environmental Microbiology, 19 (3), 878-893. doi:10.1111/1462-2920.13617

Lam, F. H., Ghaderi, A., Fink, G. R., \& Stephanopoulos, G. (2014). Biofuels. Engineering alcohol tolerance in yeast. Science, 346 (6205), 71-75. doi:10.1126/science.1257859

Li, L., Ye, Y., Pan, L., Zhu, Y., Zheng, S., \& Lin, Y. (2009). The induction of trehalose and glycerol in Saccharomyces cerevisiae in response to various stresses.Biochemical and Biophysical Research Communications, 387 (4), 778-783. doi:10.1016/j.bbrc.2009.07.113

Litsios, A., Ortega, A. D., Wit, E. C., \& Heinemann, M. (2018). Metabolic-flux dependent regulation of microbial physiology. Current Opinion in Microbiology, 42 , 71-78. doi:10.1016/j.mib.2017.10.029

Liu, C. G., Xiao, Y., Xia, X. X., Zhao, X. Q., Peng, L., Srinophakun, P., \& Bai, F. W. (2019). Cellulosic ethanol production: Progress, challenges and strategies for solutions.Biotechnology Advances, 37 (3), 491504. doi:10.1016/j.biotechadv.2019.03.002

Muhlhofer, M., Berchtold, E., Stratil, C. G., Csaba, G., Kunold, E., Bach, N. C., . . Buchner, J. (2019). The Heat Shock Response in Yeast Maintains Protein Homeostasis by Chaperoning and Replenishing Proteins. Cell Reports, 29 (13), 4593-4607 e4598. doi:10.1016/j.celrep.2019.11.109

Olsen, L. F., Stock, R. P., \& Bagatolli, L. A. (2020). Glycolytic oscillations and intracellular K(+) concentration are strongly coupled in the yeast Saccharomyces cerevisiae. Archives of Biochemistry and Biophysics, 681 , 108257. doi:10.1016/j.abb.2020.108257 
Panda, S. (2016). Circadian physiology of metabolism. Science, 354 (6315), 1008-1015. doi:10.1126/science.aah4967

Papagiannakis, A., Niebel, B., Wit, E. C., \& Heinemann, M. (2017). Autonomous Metabolic Oscillations Robustly Gate the Early and Late Cell Cycle. Molecular cell, 65 (2), 285-295. doi:10.1016/j.molcel.2016.11.018

Patnaik, P. R. (2003). Oscillatory metabolism of Saccharomyces cerevisiae: an overview of mechanisms and models. Biotechnology Advances, 21 (3), 183-192. doi:10.1016/S0734-9750(03)00022-3

Puligundla, P., Smogrovicova, D., Obulam, V. S., \& Ko, S. (2011). Very high gravity (VHG) ethanolic brewing and fermentation: a research update. Journal of Industrial Microbiology $\& 3$ Biotechnology, 38 (9), 1133-1144. doi:10.1007/s10295-011-0999-3

Richard, P. (2003). The rhythm of yeast. FEMS Microbiology Reviews, 27 (4), 547-557. doi:10.1016/S01686445(03)00065-2

Thoke, H. S., Olsen, L. F., Duelund, L., Stock, R. P., Heimburg, T., \& Bagatolli, L. A. (2018). Is a constant low-entropy process at the root of glycolytic oscillations? Journal of Biological Physics, 44 (3), 419-431. doi:10.1007/s10867-018-9499-2

Tu, B. P., Mohler, R. E., Liu, J. C., Dombek, K. M., Young, E. T., Synovec, R. E., \& McKnight, S. L. (2007). Cyclic changes in metabolic state during the life of a yeast cell.Proceedings of the National Academy of Sciences of the United States of America, 104 (43), 16886-16891. doi:10.1073/pnas.0708365104

Udom, N., Chansongkrow, P., Charoensawan, V., \& Auesukaree, C. (2019). Coordination of the Cell Wall Integrity and High-Osmolarity Glycerol Pathways in Response to Ethanol Stress in Saccharomyces cerevisiae. Applied and Environmental Microbiology, 85 (15). doi:10.1128/AEM.00551-19

Wang, L., Zhao, X. Q., Xue, C., \& Bai, F. W. (2013). Impact of osmotic stress and ethanol inhibition in yeast cells on process oscillation associated with continuous very-high-gravity ethanol fermentation. Biotechnology for Biofuels, 6 (1), 133. doi:10.1186/1754-6834-6-133

\section{Figure legends}

Figure 1. The profiles of biomass, cell viability, living cells (A) and residual glucose, ethanol, glycerol (B) as a sinusoidal function with different phases $(0$ or $\pi)$ in an oscillation period. P1-5 represent five sampling points at the beginning (P1), bottom (P2), middle (P3), top (P4), and end (P5) of the biomass oscillation period.

Figure 2 . Statistical analysis for differentially expressed genes (DEGs) according to $\log _{2}$ ratio[?]1 or [?]-1. Comparison between adjacent samples (left) and samples P2-5 to P1 (right).

Figure 3 . Integrated view of metabolome and transcriptome in central carbon metabolism pathways for neighboring samples. The gene expression and metabolite pool were marked as squares and circles, respectively. Red, green, and yellow represented up-regulation, down-regulation and no significant changes between two samples. Refer to Figure S3 for more detail of the metabolites and enzymes shown.

Figure 4. The concentrations of main intracellular metabolites (A) and the gene expression level (B) as a function of time in an oscillation period, including the main pathways of carbon metabolism, such as EMP (Red), Ethanol production (Pink), TCA (Yellow), Glycerol generation (Purple), Trehalose generation (Green), PPP (Blue) and other related factors (Black).

Figure 5. The expression level of genes related to stress responses.

Figure 6. Analysis of DEGs in the synthesis of ribosome subunits. The DEGs between (A) P2/P1, (B) P3/P2, (C) P4/P3, (D) P5/P4, and (E) the sin curve of DEGs total counts. 


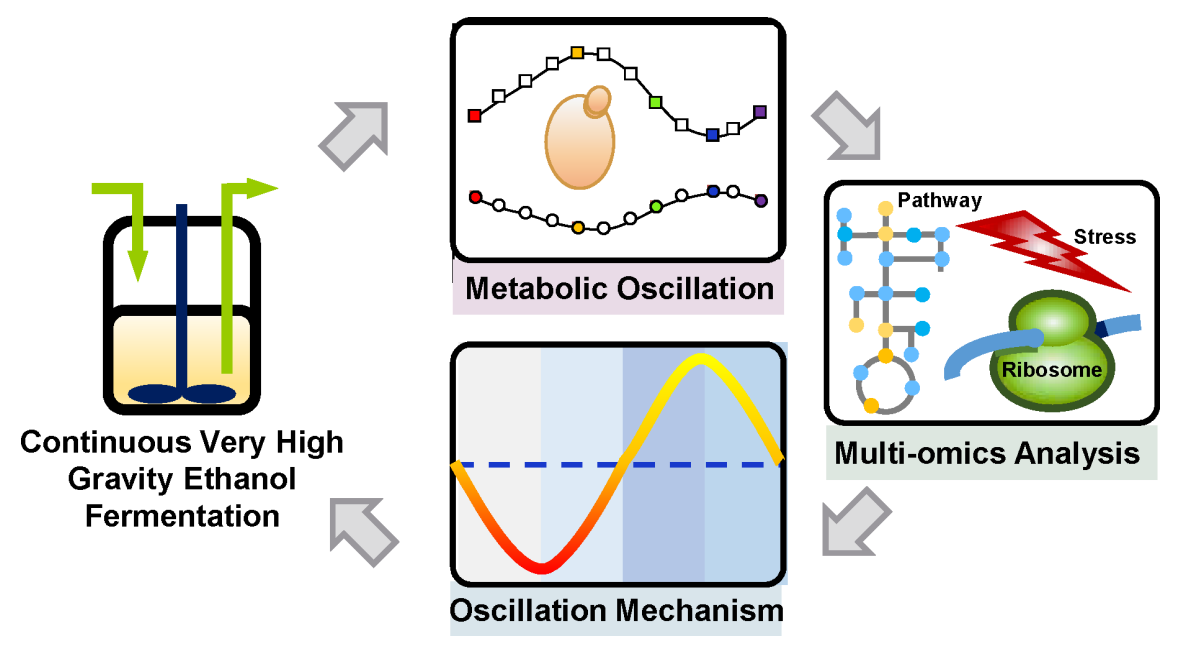

Hosted file

200615-Figures of manuscript-by LIU.docx available at https://authorea.com/users/333761/ articles/459841-omics-analysis-reveals-the-mechanism-of-metabolic-oscillation-duringcontinuous-vhg-ethanol-fermentation-by-saccharomyces-cerevisiae 